\title{
Direct evidence for the inverted band structure of HgTe
}

\author{
N. Orlowski and J. Augustin \\ Institut für Physik, Humboldt-Universität zu Berlin, Invalidenstrasse 110, D-10115 Berlin, Germany \\ Z. Gołacki \\ Institute of Physics, Polish Academy of Sciences, Al. Lotnikow 32/46, 02-668 Warsaw, Poland \\ C. Janowitz and R. Manzke \\ Institut für Physik, Humboldt-Universität zu Berlin, Invalidenstrasse 110, D-10115 Berlin, Germany
}

(Received 10 November 1999)

\begin{abstract}
Angular-resolved photoemission measurements of the nonpolar (110)-cleavage face of HgTe single crystals have been performed along the $\Sigma$ line to determine details of the band structure near the valence band maximum (VBM). Three bands are observed between VBM and $1 \mathrm{eV}$ binding energy, instead of the two observed for a positive energy gap semiconductor CdTe. Their energy separations and positions relative to the Fermi energy are investigated at the $\Gamma$ point and at slightly off-normal emission, applying room and low temperature of $40 \mathrm{~K}$. In contrast to the heavily debated results of HgSe [K.-U. Gawlik et al., Phys. Rev. Lett. 78, 3165 (1997)] the clear observations for HgTe are consistent with the model of an inverted band structure, reflecting a semiconductor with a negative band gap.
\end{abstract}

The discovery of a positive fundamental band gap on $\mathrm{HgSe}(100)$ by a combination of photoemission and inverse photoemission by Gawlik et al. ${ }^{1}$ has raised the general question of the validity of the inverted band structure model for mercury based II-VI compounds. Since this model has so far been underlying the physics of these materials, these findings have stimulated new discussions ${ }^{2}$ and investigations, e.g., of magnetooptic techniques. ${ }^{3}$ Furthermore, in a very recent first principle local-density approximation (LDA) calculation within the GW approximation of $\mathrm{HgSe}$, the hitherto accepted view for the sequence of critical points at the $\Gamma$ point of $\Gamma_{8}$, $\Gamma_{6}$, and $\Gamma_{7}$ has been found to be changed to $\Gamma_{8}, \Gamma_{7}$, and $\Gamma_{6}^{4}$. Their negative gap of about $0.5 \mathrm{eV}$ should have been observed by photoemission. ${ }^{1}$

All these contradicting findings for $\mathrm{HgSe}$ make the determination of the valence band structure of $\mathrm{HgTe}$ worthwhile, the prototype of the mercury based II-VI semiconductors. The most direct experimental access to the electronic band structure is enabled by photoemission, in particular when it is performed with high angle and energy resolution and at low temperature. The investigation of $\mathrm{HgTe}$ instead of $\mathrm{HgSe}$ is advantageous in several respects: (i) The HgTe (110) surface is nonpolar, i.e., it exhibits charge neutrality and cleaves reproducibly showing sharp $(1 \times 1)$ low-energy electron diffraction (LEED) patterns. (ii) Due to the fact that the uppermost valence band region is mainly made up by chalcogene states, the larger spin-orbit splitting of Te (about $0.9 \mathrm{eV}$ instead of $0.3 \mathrm{eV}$ for $\mathrm{Se}$ ) makes an identification of additional bands in this energy region much easier. (iii) Moreover, the clearly spin-orbit split Te bands present themselves-like a finger print-for a direct comparison with CdTe, a II-VI semiconductor with a well-accepted positive band gap.

HgTe has been extensively studied by transport, ${ }^{5}$ optical, ${ }^{6}$ and magnetooptical ${ }^{7}$ measurements. These have established the inverted band structure model for the $\mathrm{Hg}$ derived II-VI semiconductors which was for the first time proposed for $\alpha$
Sn. ${ }^{8}$ In contrast to this, there exists up to now only a few photoemission studies concerning the valence band structure of $\mathrm{HgTe},{ }^{9,10}$ which, due to limited experimental resolution, cannot address the above questions. In this paper, we present angle-resolved photoemission (ARPES) spectra of the valence band of $\mathrm{HgTe}(110)$ performed with high resolution at room and cryogenic temperatures. At first, the valence band maximum (VBM) is determined from photon energy dependent measurements at normal emission. The number, sequence, and energy of the bands at $\Gamma$ is derived from a density of states model, applying Gaussian profiles and the Fermi-Dirac distribution. Then, the $\Gamma$-point spectrum is compared to slightly off-normal spectra and to similar spectra of CdTe. This detailed analysis will reveal the plain difference between HgTe and semiconductors with a positive band gap, such as CdTe.

The HgTe crystals were grown by a modified Bridgman technique at the Polish Academy of Sciences in Warsaw. Quality and orientation were controlled by $\mathrm{x}$-ray diffraction. The crystals were cut into pieces of $3 \times 3 \times 2 \mathrm{~mm}^{3}$ size and were cleaved in an ultrahigh vacuum by the anvil and wedge technique along the (110) surface, at liquid nitrogen temperature with flat surfaces. As was pointed out by Yu et al., ${ }^{11}$ extrinsic surface core level shifts at the spin-orbit split $\mathrm{Hg} 5 \mathrm{~d}$ bands can be observed in photoemission spectra in the case of poor cleavage. We never observed this effect in our spectra, so it is concluded that our samples always had good surface quality. This was on occasion also confirmed by the observation of sharp $(1 \times 1)$ LEED patterns.

The photoemission measurements were performed at room temperature and at $40 \mathrm{~K}$. We applied synchrotron radiation in the range $h \nu=10-30 \mathrm{eV}$ from the DORIS III storage ring at the HONORMI beamline of HASYLAB in Hamburg, and at the 3m-NIM-1 beamline of the BESSY I storage ring in Berlin, both equipped with a $3 \mathrm{~m}$ normal-incidence monochromator, and electron spectrometers with high en- 

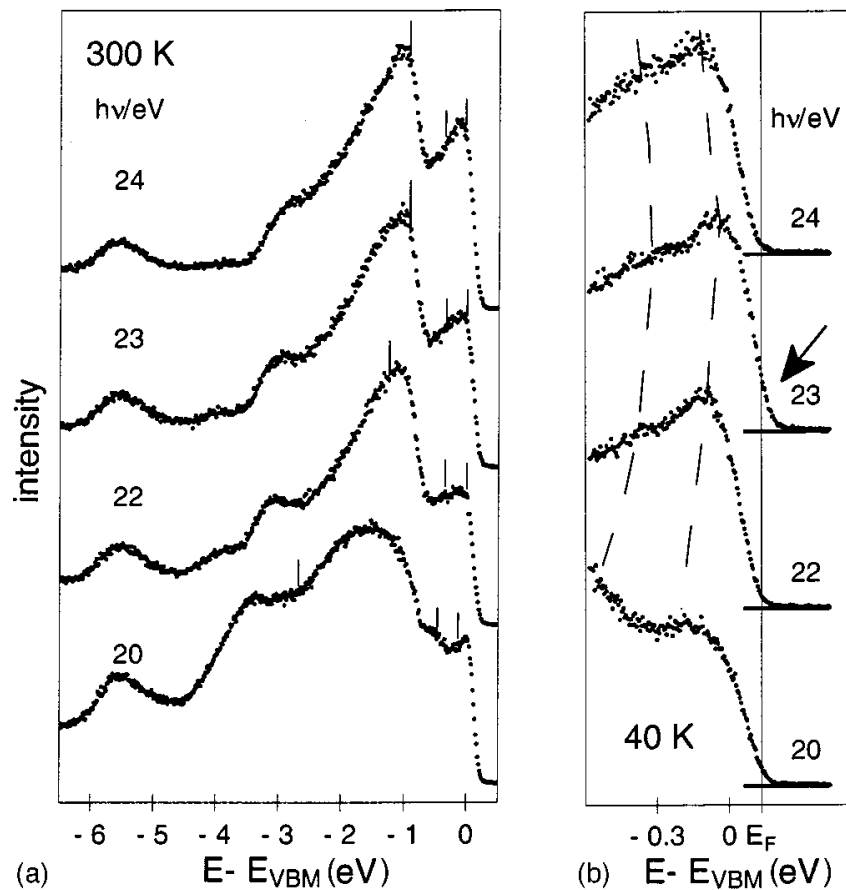

FIG. 1. Energy distribution curves in normal emission of the photon energy range $h \nu=20-24 \mathrm{eV}$ at $300 \mathrm{~K}$ (left panel) and $40 \mathrm{~K}$ (right panel). The energies of emissions due to the heavy/light hole and split-off band (see text) as determined by a fit procedure are marked by ticklines.

ergy and angular resolution consisting of a hemispherical electron energy analyzer mounted on a two-axes goniometer. The overall energy resolution was below $100 \mathrm{meV}$, the angular resolution depending on the spectrometer was in the range $\Delta \vartheta=0.5^{\circ}-1^{\circ}$. This energy resolution was found sufficient to resolve the intrinsic width of the structures. The Fermi energy was determined by photoemission from a polycrystalline gold film evaporated prior to cleavage onto a plate of copper attached to the sample holder.

Figure 1 shows a selection of energy distribution curves (EDC's) taken in normal emission in the photon energy range $h \nu=20-24 \mathrm{eV}$, i.e., along the $\Sigma$ line of the bulk Brillouin zone, at room temperature (left panel) and at $40 \mathrm{~K}$ (right panel). The vector potential of the incoming synchrotron radiation was in the mirror plane of the crystal surface $\overline{\Gamma X^{\prime}}$ and had an angle of $45^{\circ}$ (left) and $38^{\circ}$ (right) with respect to the surface normal. The binding energy is referred to the valence band maximum, whose determination is discussed below.

The valence band maximum at the $\Gamma$ point is given by the photoemission structure with minimum binding energy in the photon energy dependent series. In accordance with Ref. 10, this is fulfilled for $h \nu=23 \mathrm{eV}$. It becomes especially clear from the series of the right panel of Fig. 1 that for $h \nu$ $=23 \mathrm{eV}$, the edge of the leading structure has the largest overlap with the Fermi energy (see the arrow). Taking a sum of Gaussian profiles superimposed on a Shirley type background $^{12}$ as a model for the spectra, in a least squares fit procedure, and taking the experimentally known spectrometer function and the Fermi-Dirac distribution into account, the exact position of the valence band maximum is obtained. The determination of the energetic positions of all photo- emission features in a similar way results in the complete experimental valence band structure of $\mathrm{HgTe}$ along the $\Sigma$ line, which will be published elsewhere. However, for the purpose of a rough overview, we show ticks in the spectra of Fig. 1 marking energy positions of the Gaussians which have to be attributed to the heavy/light hole and split-off band, but which, due to the fit of the whole spectrum, need not necessarily coincide with emission maxima, especially for higher binding energies, where also additional emission due to mnklapp bands occurs. The dispersion of the uppermost bands becomes more obvious in the right panel of Fig. 1, as is suggested by the dashed lines. ${ }^{13}$

At the $\Gamma$ point, i.e., the spectrum at $h \nu=23 \mathrm{eV}$, two distinct structures are seen near VBM (Fig. 1, left panel) which are separated by about $1 \mathrm{eV}$. These can be attributed to the spin-orbit split $\Gamma_{8}$ and $\Gamma_{7}$ levels. For a detailed analysis, the $\Gamma$-point spectrum is also shown in Fig. 2(c) in comparison to the according $\Gamma$-point spectrum of CdTe. In contrast to $\mathrm{CdTe}(110)$, (from Ref. 14) the leading structure of $\mathrm{HgTe}$ reveals an asymmetric shape. As a result, the distinct minimum between $\Gamma_{8}$ and $\Gamma_{7}$ of CdTe is found to be filled up for HgTe. This suggests immediately the existence of a third emission maximum in the binding energy region between the spin-orbit split peaks. Quantitatively, it is determined by the fit procedure, whose result is also shown in Fig. 2(c). The peak $\Gamma_{7}$ is located $0.91 \mathrm{eV}$ below the valence band maximum $\Gamma_{8}$, giving the same spin-orbit splitting, $E\left(\Gamma_{8}\right)-E\left(\Gamma_{7}\right)$, as for CdTe. ${ }^{4}$ This similarity is not unexpected because in both substances this energy region is build up by states of Te5p electrons. Between the spin-orbit split peaks, we derive for $\mathrm{HgTe}$ an additional peak located 0.33 $\mathrm{eV}$ below the valence band maximum. This is absent in CdTe and has to be interpreted as the $\Gamma_{6}$ level according to the model of the inverted band structure. Thus, the negative fundamental band gap, $E\left(\Gamma_{6}\right)-E\left(\Gamma_{8}\right)$, of $\mathrm{HgTe}$, is at room temperature $0.33 \mathrm{eV}$.

The existence of the additional peak due to the negative band gap is further supported by off-normal spectra. In Fig. 2(a) and 2(d), we compare slightly off-normal spectra of the $\overline{\Gamma X^{\prime}}$ direction of the surface Brillouin zone. For $\operatorname{HgTe}(110)$, the emission angle is $\vartheta=2.5^{\circ}$, for $\operatorname{CdTe}(110), \vartheta=2^{\circ}$. This is equivalent to a $k_{\|}$wave vector of about $0.095 \AA^{-1}$ and $0.08 \AA^{-1}$, respectively, i.e., about $5 \%$ of the bulk Brillouin zone. In the case of CdTe, the spectra show, besides some small dispersion effects, almost no difference. In contrast to this, for HgTe the additional band due to the asymmetric structure at $\vartheta=0^{\circ}$ develops to a distinct maximum at $\vartheta$ $=2.5^{\circ}$, the $\Gamma_{6}$ light hole band. Here, it has to be mentioned that this $\Gamma_{6}$ emission at a small off-normal angle of emission is only observed if the direction of emission falls between the surface normal and the incidence direction of the light while it is suppressed on the other side of the surface normal.

In order to investigate the band gap of $\mathrm{HgTe}$ in more detail, a $\Gamma$-point spectrum $(h \nu=23 \mathrm{eV})$ of $40 \mathrm{~K}$ is analyzed in a highly resolved binding energy range, see Fig. 3. At low temperature, the $\Gamma_{6}$ band appears more clearly. Analogous to the corresponding room temperature spectrum [Fig. 2(c)], we applied the same fit procedure. For a realistic model it was also necessary to take the slope of the $\Gamma_{7}$ split-off band into account. The corresponding parameters are taken from the room temperature result but they were not subject to varia- 


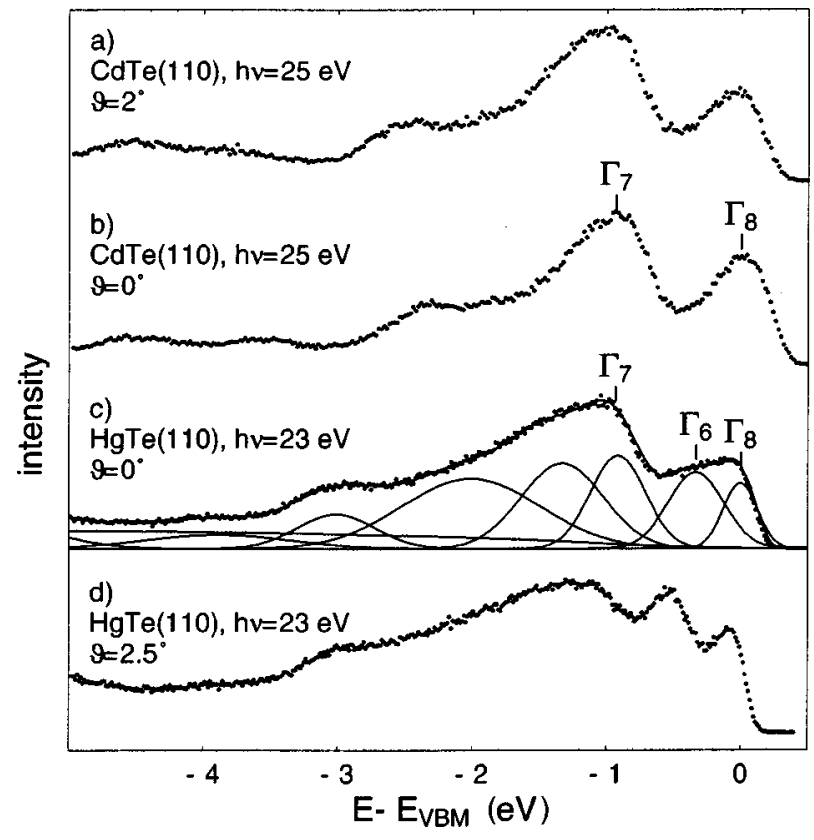

FIG. 2. EDC's at $300 \mathrm{~K}$ in normal and slightly off-normal emission for $\mathrm{HgTe}(110)$ and $\mathrm{CdTe}(110)$ at $h \nu=23 \mathrm{eV}$ and $h \nu=25 \mathrm{eV}$, respectively. For $\mathrm{HgTe}$ at $\vartheta=0^{\circ}$, the result of a least squares fit is shown with the upper valence band peaks being explicitly assigned.

tion. The result, i.e., the contributions of the $\Gamma_{8}, \Gamma_{6}$, and $\Gamma_{7}$ emissions as well as the Shirley type background ( $\mathrm{Sh}$ ), is shown in Fig. 3. Note that the fit to the experimental spectrum is only satisfying if, in addition, a Fermi-Dirac distribution is explicitly taken into account. From this, the negative band gap is $E_{0}=-0.30 \mathrm{eV}$. In order to illustrate the effect of the Fermi-Dirac distribution on the spectrum, Fig. 3 also shows the fit curve that would be achieved when the Fermi edge is neglected (dashed curve). There exists only a very small photon energy and emission angle range where the effect of the Fermi-Dirac distribution can be observed. This becomes, for instance, clear from Fig. 1 (right panel) where the overlap of the photoemission onset with the Fermi energy is marked by an arrow.

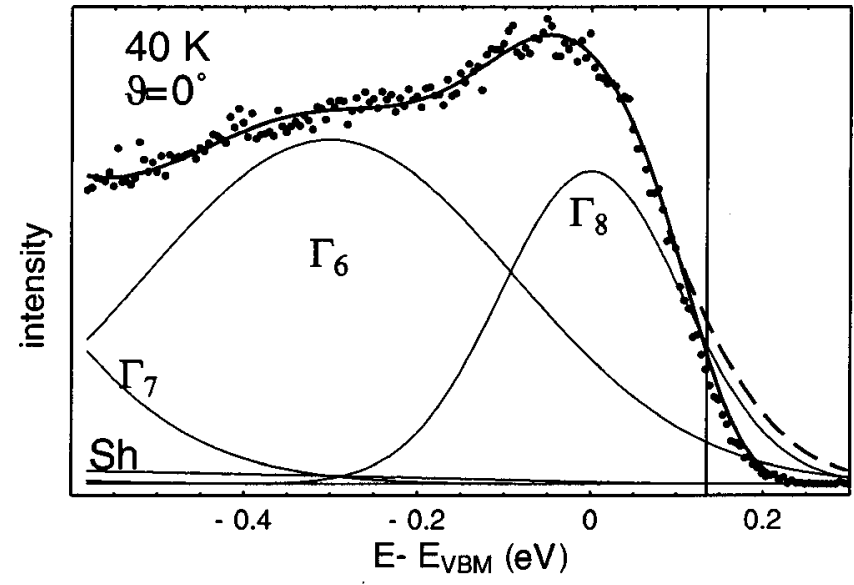

FIG. 3. Energy distribution curve of $\mathrm{HgTe}(110)$ at $h \nu=23 \mathrm{eV}$ in normal emission at $40 \mathrm{~K}$, together with a least squares fit (thick line) as a sum of Gaussians and Shirley background (thin lines). The dashed line shows the results if the Fermi-Dirac distribution (Fermi edge) is neglected.
TABLE I. Band gaps $E_{0}=E\left(\Gamma_{6}\right)-E\left(\Gamma_{8}\right)$ at $40 \mathrm{~K}$ and at $300 \mathrm{~K}$, and spin-orbit splitting $\Delta=E\left(\Gamma_{8}\right)-E\left(\Gamma_{7}\right)$ of $\mathrm{HgTe}$ as determined in this study, compared to other experimental and theoretical results.

\begin{tabular}{cccc}
\hline \hline & this study & exp. $^{\text {a }}$ & theory $^{\mathrm{b}}$ \\
\hline$E_{0}(40 \mathrm{~K}) / \mathrm{eV}$ & $-0.29 \pm 0.02$ & -0.28 & -0.61 \\
$E_{0}(300 \mathrm{~K}) / \mathrm{eV}$ & $-0.32 \pm 0.03$ & -0.14 & \\
$\Delta / \mathrm{eV}$ & $0.91 \pm 0.02$ & 1.08 & 0.78 \\
\hline \hline
\end{tabular}

a Band gaps $E_{0}$ from optical data (Ref. 15), spin-orbit splitting $\Delta$ from electroreflectance data (Ref. 16).

${ }^{\mathrm{b}} \mathrm{Ab}$ initio theory from Ref. 17.

As a result of the preceding discussion, the negative band gap of $\mathrm{HgTe}$ is worked out at room temperature and at $40 \mathrm{~K}$. Being aware that results of least squares fits are in principle not unambiguous, several fits have been performed. Mean values for the band gap are $E_{0}(300 \mathrm{~K})=-0.32 \pm 0.03 \mathrm{eV}$ and $E_{0}(40 \mathrm{~K})=-0.29 \pm 0.02 \mathrm{eV}$. The gap values together with the spin-orbit splitting are compared to previous results in Table I. For $40 \mathrm{~K}$, the band gap is in good agreement with the value derived from various optical and magnetooptical measurements (see the compilation of Ref. 15), where $E_{0}(T=40 \mathrm{~K})=-0.28 \mathrm{eV}$ was obtained. Our room temperature value, however, is on a first sight striking. Compared to the very different room temperature value of $0.14 \mathrm{eV},{ }^{15}$ the temperature variation of the photoemission value is not only distinctly weaker, it points also to a temperature coefficient of the gap that has the opposite sign, though we note that both values coincide within the error bars. Hansen et al. ${ }^{15}$ derived $d\left(E\left(\Gamma_{6}\right)-E\left(\Gamma_{8}\right)\right) / d T=5.35 \times 10^{-4} \mathrm{eV} / \mathrm{K}$, in contrast to the value of the presented photoemission data of $d\left(E\left(\Gamma_{6}\right)-E\left(\Gamma_{8}\right)\right) / d T=-1.2 \times 10^{-4} \mathrm{eV} / \mathrm{K}$. In other words, photoemission proposes that the energy separation between the $\Gamma_{6}$ and $\Gamma_{8}$ levels, i.e., the negative gap, increases with increasing temperature. This behavior is opposite to that of positive gap zinc-blende-type semiconductors. Regarding, however, the temperature dependence of the hybridization gap, due to the variation of the lattice parameter, such an opposite behavior is not unexpected. From an estimation the energy of the critical points $\Gamma_{8}$ and $\Gamma_{6}$ increases and decreases, respectively, with increasing lattice parameter. But due to the fact that for negative gap material, $\Gamma_{8}$ is above $\Gamma_{6}$, this means an increase of the gap, in line with the photoemission result. A positive temperature dependence of the gap for a negative gap material would, on the contrary, not be understandable. Nevertheless, this has to be quantitatively supported by a temperature dependent quasiparticle band structure calculation of $\mathrm{HgTe}$.

The valence band maximum, i.e., the center of gravity of the leading peak $\Gamma_{8}$, is found about $0.1 \mathrm{eV}$ below the Fermi energy (see Fig. 3). On the other hand, the onset of the spectra reveals at $\Gamma$ a Fermi-Dirac cutoff. This cutoff is an additional strong hint of the inverted band structure, where the gap at $E_{F}$ should be zero. Thus, the difference between VBM and $E_{F}$, which is definitely not due to experimental uncertainties, points to a partly occupied conduction band. The mercury based II-VI compounds are known to tend towards self-intercalation effects, generating a small amount of excess charges. The question is raised of whether this could be 
observed. The band mass of the conduction band is 0.03 electron masses. ${ }^{18}$ Compared to the band mass of 0.4 electron masses of the $\Gamma_{8}$ heavy hole band, ${ }^{18}$ the value of the conduction band and hence the density of states is extremely small. Our simulations showed that the effect is too weak to be observed in the photoemission spectra, besides the shift of $E_{F}$.

In conclusion, we have derived direct experimental evidence of the validity of the inverted band structure model for HgTe by angle-resolved photoelectron spectroscopy. The negative fundamental energy gap $E_{0}$ is $0.32 \mathrm{eV}$ at room temperature, and the zero gap behavior at $\Gamma_{8}$ is manifested by the observation of a Fermi edge. The temperature dependence of the negative gap is negative. This result of the direct analysis of the wave vector dependent density of states given by our photoemission spectra is in contradiction to previous data derived from magnetooptical measurements. ${ }^{15}$ Although a negative temperature dependence of $E_{0}$ is reasonable for semiconductors with inverted band structure, it asks for quantitative theoretical support, e.g., by taking the influence of the temperature dependent interatomic interactions explicitly into account.

We would like to thank Professor B. A. Orlowski (Institute of Physics of the Polish Academy of Sciences, Warsaw) for supplying HgTe single crystals, and Professor M. Skibowski (University of Kiel) and his group for technical support at the HONORMI beamline. Furthermore, we thank the staff of HASYLAB and BESSY. This work received funding from the BMBF under Projects No. 05622 KHA and No. 05 SB8 KH1(0).
${ }^{1}$ K.-U. Gawlik, L. Kipp, M. Skibowski, N. Orlowski, and R. Manzke, Phys. Rev. Lett. 78, 3165 (1997).

${ }^{2}$ T. Dietl, W. Dobrowolski, J. Kossut, B. J. Kowalski, W. Szuszkiewicz, Z. Wilamowski, and A. M. Witowski, Phys. Rev. Lett. 81, 1535 (1998); K.-U. Gawlik, L. Kipp, M. Skibowski, N. Orlowski, and R. Manzke, ibid. 81, 1536 (1998).

${ }^{3}$ M. von Truchseß, A. Pfeuffer-Jeschke, C. R. Becker, G. Landwehr, and E. Batke, Phys. Rev. B 61, 1666 (2000).

${ }^{4}$ M. Rohlfing and S. G. Louie, Phys. Rev. B 57, R9392 (1998).

${ }^{5}$ R. Piotrzkowski, S. Porowski, Z. Dziuba, J. Ginter, W. Giriat, and L. Sosnowski, Phys. Status Solidi 8, K135 (1965).

${ }^{6}$ W. Szuszkiewicz, Phys. Status Solidi B 79, 691 (1977).

${ }^{7}$ S. H. Groves, R. N. Brown, and C. R. Pidgeon, Phys. Rev. 161, 779 (1967).

${ }^{8}$ S. Groves and W. Paul, Phys. Rev. Lett. 11, 194 (1963).

${ }^{9}$ J. A. Silberman, D. Laser, C. K. Shih, D. J. Friedman, I. Lindau, W. E. Spicer, and J. A. Wilson, J. Vac. Sci. Technol. A 3, 233 (1985); C. K. Shih, J. A. Siberman, A. K. Wahi, G. P. Carey, I.
Lindau, W. E. Spicer, M. A. Berding, and A. Sher, ibid. 5, 3026 (1987).

${ }^{10}$ M. Banouni, M. Nasser, and G. Leveque, J. Cryst. Growth 159, 736 (1996).

${ }^{11}$ Xiaohua Yu, L. Vanzetti, G. Haugstad, A. Raisanen, and A. Franciosi, Surf. Sci. 275, 92 (1992).

${ }^{12}$ D. A. Shirley, Phys. Rev. B 5, 4709 (1972).

${ }^{13}$ For photon energies less than about $20 \mathrm{eV}$, the leading photoemission edge at VBM is increasingly built up by emission from a surface state, which will be published elsewhere.

${ }^{14}$ C. Janowitz, L. Kipp, R. Manzke, and B. A. Orlowski, Surf. Sci. 231, 25 (1990).

${ }^{15}$ G. L. Hansen, J. L. Schmitt, and T. N. Casselman, J. Appl. Phys. 53, 7099 (1982).

${ }^{16}$ A. Moritani, K. Taniguchi, C. Hamaguchi, and C. Nakai, J. Phys. Soc. Jpn. 34, 79 (1973).

${ }^{17}$ D. Vogel, Ph.D. thesis, Universität Münster, 1998.

${ }^{18}$ Y. Guldner, C. Rigaux, M. Grynberg, and A. Mycielski, Phys. Rev. B 8, 3875 (1973). 